\title{
Analytical estimation of the reliability of wire rope based on cumulative damage
}

\author{
Achraf. WAHID ${ }^{1,2^{*}}$, Nadia MOUHIB ${ }^{2}$, Fatima. SABAH ${ }^{1}$, Hamid CHAKIR $^{1}$, Mohamed EL GHORBA $^{2}$ \\ ${ }^{1}$ Faculté des Sciences Ben M'sik Avenu Driss El harti, B.P.7955, Sidi Othmane Casablanca, Maroc \\ ${ }^{2}$ Laboratoire de Contrôle et Caractérisation Mécanique des Matériaux et des structures, B.P 8118, Oasis-Route El Jadida - \\ ENSEM / Casablanca, Maroc \\ *E-MAIL:Achraf.wahid90@hotmail.com
}

\begin{abstract}
The main objective of this manuscript is the elaboration of a behavior model of the cable system, designed to the prediction of its damage from the behavior of its components (wires, strands). This multifaceted scale is based primarily on the results experiments of the central core and helical strands components. At first, a resolution numerical probabilistic equation for the different configurations was established by the programming the system resolution scheme obtained, subsequently, each damage curves obtained, corresponding to a particular configuration has been interpreted independently.
\end{abstract}

Keywords: Damage; reliability; analytical model; strand; cable;

\section{Introduction}

The wire rope is widely used in industrial production and daily life [1] such as the aircraft industry, the systems of lifting, the electric lines...Etc. Therefore, the wire rope suffers from the continuous aggression of the environment[2] (Urban, industrial, marine... etc.), and very different conditions of use according to the application considered, The effects of these factors are manifested through several phenomena .However, the wire rope deteriorates with age, loading, and environmental effects, and they experience damage during incidents [3]. For example, the strands used in bridge cables tend to corrode and break when in service[4-3], thus reduction in the resistance capacity of the cable as a function of time, sometimes leading to partial rupture[5] at the same time in the process of fracture failure process of wire ropes, the number of broken wires increases gradually, until the whole wire rope breaks [1]. A great deal of research and development has been carried out over the years to better understand the physical behavior of such systems. Most of this work has focused on the impact of wire fracture on the behavior of multi-wire ropes or strands/cables. The industrialists always tend to explore new grounds in order to discover effective control tools that can determine the state of the life of a structure with minimal time and low cost. It is in this context where our work is written which aims to predict the damage of the cable from its components (wire, strands) for that a multitude of function has been developed which is intended to calculate the damage of the systems complex, whose functional description is translated by a block system.

\section{Multiscale approach of a wire rope metallic}

Generally speaking, the wire rope is made of several strands laid helically around a central core, in one or multiple layers. The strand is itself composed of several steel wires arranged regularly in a helix around a central core, in one or more superposed layers. They are characterized by a hierarchical structure; hence the use of scale transition models to switch from the behavior of the son to that of the cables.

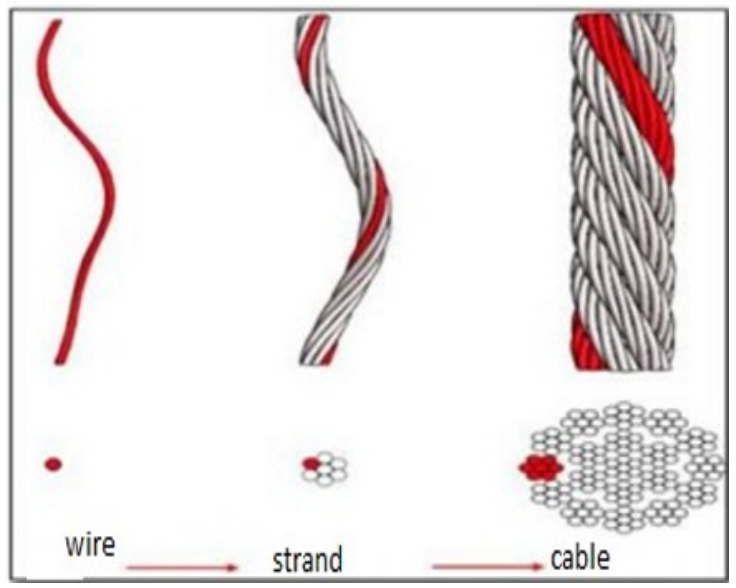

Figure 1. Schematic representation of the three scales (wires, strand and cable).

\subsection{Quantification of static damage}

The model of static damage consists in determining the evolution of the force according to the level of damage. The static damage is then quantified by the variable Ds expressed by:

$$
D_{s}=\frac{1-\frac{F_{u r}}{F_{u}}}{1-\frac{F a}{F u}}
$$

with:

Fu: Value of the ultimate force in the initial state;

Fur: Value of the ultimate force for different levels of damage;

Fa: Force just before the break. 
In our paper, we based on the equation (1) to follow the damage of strand extracted from steel wire rope. For this, static tests were performed on artificially damaged specimens at different levels of damage by cutting some wires $(14 \%, 28 \%, 42 \%, 57 \%$ and $71 \%$ broken wires).

$\beta$ is the fraction of life ;

$\begin{array}{lll}>\text { For } \beta=0 \text { (undamaged strand) } & \\ \text { we have For } \beta=1 & \text { Fur }=\mathrm{Fu} & \mathrm{D}=0 \text {. } \\ \text { we have } \quad \text { Fur }=\mathrm{Fa} & \text { so } & \mathrm{D}=1\end{array}$

The equation $\left(\mathrm{n}^{\circ} 1\right)$ represents the obtained damage by the strength residual method. As well, we obtain the reliability by the relation:

$$
R(\beta)=1-D(\beta)
$$

\section{The reliability of composed systems}

The reliability of a material is a statistic parameter. It represents the probability of the survival of this material. In the case of the multi-component system, the failure of the system depends on the failure of a number of its components. The cable concerned by this study can be considered as a block system consisting of three layers (the central core, the inner layer and the outer layer) arranged in series, each layer and itself consists of a set of strands arranged in parallel. Each strand consists of several twisted wires arranged in turn logic-Majority system.

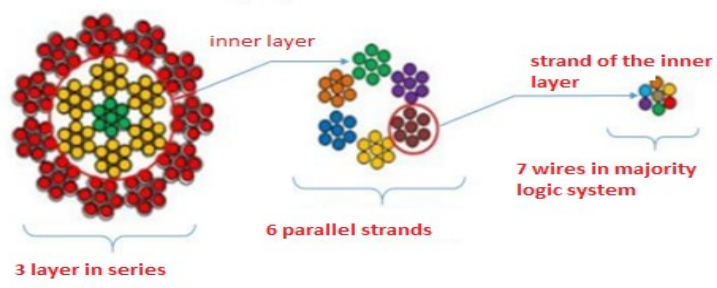

Figure 2. Diagram showing the system blocks of the non-rotating rope $19 \times 7$

In the case of our system, the cable is considered to be a mixed system. Therefore, the choice is justified by:

- A broken wire does not cause the failure of the strand, however against a number of broken wires, the strand is declared failure. According to the experiments that were solicited in the work of BOUDLAL [6] and following the results of MOUHIB [7], our strand is declared failure from five broken wires according to the change of the curve of damage. Which allowed us to exploit the following equation (3):

$$
R=\sum_{j=m^{\prime}}^{n '} C_{n^{\prime}}^{m^{\prime}} R^{j}(1-R)^{n^{\prime}-j}
$$

With:

$\mathbf{n}^{\prime}$ : the number of wires in the strand $\mathbf{m}^{\prime}$ : minimal threshold of the number of functional wires .
This formula takes into account the state of degradations of a strand according to the numbers of the wires broken.

if $\mathbf{m}^{\prime}=\mathbf{n}^{\prime}$ we find according the equation of system series .

$$
R=R^{n^{\prime}}
$$

If the failure criterion corresponds to a single broken wire, that is to say that for $\mathbf{m}^{\prime}=\mathbf{1}$.

$$
\begin{aligned}
& R=\sum_{j=1}^{n^{\prime}} C_{n^{\prime}}^{m^{\prime}} R^{j}(1-R)^{n^{\prime}-j} \\
& =\sum_{j=0}^{n^{\prime}} C_{n^{j}}^{j} R^{j}[1-R]^{n^{\prime}-j}-C_{n^{\prime}}^{0} R^{0}(1-R)^{n^{\prime}-0} \\
& =[R+1-R]^{n^{\prime}-}-[1-R]^{n,} \\
& =1-[1-R]^{n^{\prime}}
\end{aligned}
$$

This is the reliability of a parallel system.

* At the level of the layer, a broken strand does not cause the failure of all the layer, which makes it possible to deduce that the strands are organized in a parallel manner, therefore:

$$
R_{\text {layer }}=1-\prod_{i=1}^{n}\left(1-R_{\text {strand }}\right)
$$

* At the level of the cable, if there is a broken layer, the system is thus failing in its entity, knowing that the central core represents $25 \%$ of the breaking strength of the cable

$$
R_{\text {cable }}=\prod_{i=1}^{m} R_{\text {klayer }}
$$

From the results found above, the cable is considered a majority logical / parallel /serial system, so the reliability function that translates our system is of the following form:

$$
R_{\text {cable }}=\prod_{k=1}^{m}\left(1-\prod_{i=1}^{n}\left(1-\sum_{1}^{n^{\prime \prime}} C_{n^{\prime}}^{n^{\prime}} R_{\text {wire }}^{j}\left(1-R_{\text {wire }}\right)^{n^{\prime}-j}\right)\right)
$$

The final equation that means our system is a series-parallel equation that takes the following form:

$$
R_{\text {cable }}=\prod_{k=1}^{m}\left(1-\prod_{i=1}^{n}\left(1-R_{\text {kstrand }}\right)\right)
$$

$\mathbf{m}$ : is the number of layers in the cables

n: number of strands in each layer

Knowing that our system is not symmetrical, that is to say, each layer consists of a number of strands different from the others,For simplification we take:

$$
R_{\text {cable }}=\left(1-\prod_{i=1}^{12}\left(1-R_{k i}\right)\right)\left(1-\prod_{i=1}^{6}\left(1-R_{k i}\right)\right)\left(1-\left(1-R_{c o r e}\right)\right)
$$

\section{Application of the proposed model on the relationship damage - reliability}


Reliability varies in the opposite direction of damage. Intuitively, there is a relationship between these two parameters equation (2), Which allows us to write:

$$
D_{\text {cable }}=1-\left(1-\prod_{i=1}^{12} D_{\text {strand }}\right)\left(1-\prod_{i=1}^{6} D_{\text {strand }}\right)\left(1-D_{\text {core }}\right)
$$

A calculation program based on equation (10) of the system has been established, whose approach is summarized in the following configurations:

\section{Configuration I :}

The failure of the layers in this configuration is as follows: We start by breaking a strand, wire by wire, followed by breaking the second strand just at side, and so on until the break of the entire outer layer, followed the inner layer and finally the central core.

\section{Configuration II:}

In this configuration the failure begins with the breaking of four wires at the same time, each strand in each layer separately, then the remaining wires are broken off one by one, strand by strand, until the total breaking of the layer concerned

\section{Configuration III:}

On the basis of the same series of rupture of the configuration II, the damage of the layers of course remains the same. However, what is changing here is the total damage cable.

This time it is considered that the wires are broken in a different way than the previous ones. the failure of this configuration begins with the breaking of a first strand of the outer layer and then of a first strand of the inner layer, then of a second strand of the outer layer followed by a second strand of the inner layer and thus immediately, until reaching the 6th strand of the latter where we touch the 7 th thread. Subsequently, the remaining 6 strands of the outer layer are broken, leaving the 7 th wire of the last strand alone. Finally, we break the central core with the two remaining wires of the inner and outer layers.

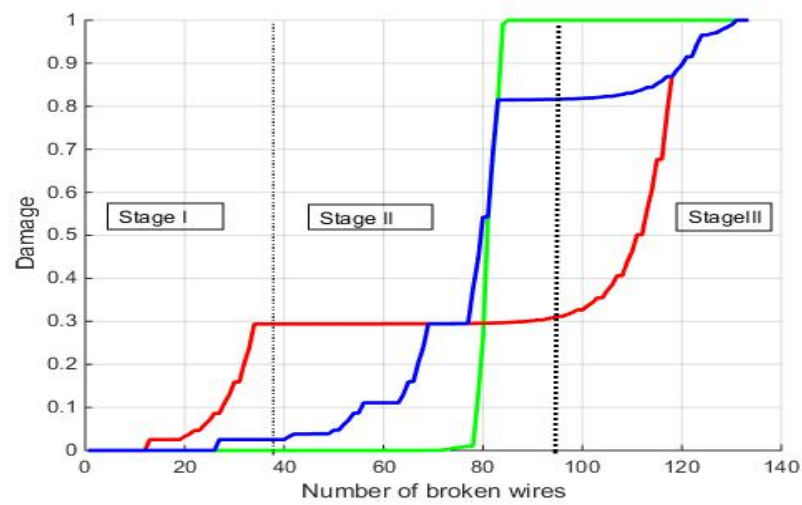

Figure 3: superposition of the damage curves of the treated cases

The figure (3) illustrate the damage evolution of the three models configuration presented in this paper, the evolution of the static damage according to the number of broken wire .according the this figure we can identify three damage stage by dint of the change of curves and we can compare the damage of the three configurations at each stage.

\section{Conclusion}

This study made it possible to carry out a multi-scale study of the mechanical behavior of the cable structure in order to ensure its industrial exploitation in optimal conditions.

As a result, criteria have been discussed that take into account the number of breakable wires tolerable during use prior to removal of the cable. In addition, we have developed a modeling to predict the resistance of a cable to various levels of damage to the constituent elements.

\section{Références}

[1]:Wenzheng, D., Baozhu, M., Zheng, X., Dazhi, C., \& Peng, W. (2017). Finite element analysis on the wire breaking rule of $1 \times 7$ IWS steel wire rope. In MATEC Web of Conferences (Vol. 108, p. 01002). EDP Sciences. [2]: Crémona, C., \& Rasson, O. (2002). Approche probabiliste de la résistance résiduelle des câbles de structures. Revue française de génie civil, 6(3), 397-407.

[3]: Qu, H., Li, T., \& Chen, G. (2017). Influence Length of Wire Fracture and Wire-to-Wire Interaction in Helically Wired Strands under Axial Loads. Journal of Bridge Engineering, 23(1), 04017110.

[4]:Betti, R., \& Yanev, B. (1999). Conditions of suspension bridge cables: New York City case study. Transportation Research Record: Journal of the Transportation Research Board, (1654), 105-112.

[5]: Singh, R. P., Mallick, M., \& Verma, M. K. (2016). Studies on failure behaviour of wire rope used in underground coal mines. Engineering Failure Analysis, 70, 290-304

[6]: Boudlal, E., Barakat, M., Mouhib, N., (...), El Ghorba, M., Ouaomar, H.Mechanical behaviour of damaged central core strand constituting a steel wire rope hoist under the effect of a static load (IJMET)

[7]: N. Mouhib, Multi-scale study of the mechanical behavior of a non-rotating lifting wire rope $(19 \times 7)$ subjected to static loads and prediction of its lifetime: experimental, analytical and numerical, Doctoral thesis, ENSEM, Hassan 2 University, Ain Chock, Casablanca, 2015 